\title{
Fine-Grained Object Recognition and Zero-Shot Learning in Remote Sensing Imagery
}

\author{
Gencer Sumbul, Ramazan Gokberk Cinbis, and Selim Aksoy ${ }^{\circledR}$, Senior Member, IEEE
}

\begin{abstract}
Fine-grained object recognition that aims to identify the type of an object among a large number of subcategories is an emerging application with the increasing resolution that exposes new details in image data. Traditional fully supervised algorithms fail to handle this problem where there is low betweenclass variance and high within-class variance for the classes of interest with small sample sizes. We study an even more extreme scenario named zero-shot learning (ZSL) in which no training example exists for some of the classes. ZSL aims to build a recognition model for new unseen categories by relating them to seen classes that were previously learned. We establish this relation by learning a compatibility function between image features extracted via a convolutional neural network and auxiliary information that describes the semantics of the classes of interest by using training samples from the seen classes. Then, we show how knowledge transfer can be performed for the unseen classes by maximizing this function during inference. We introduce a new data set that contains 40 different types of street trees in 1-ft spatial resolution aerial data, and evaluate the performance of this model with manually annotated attributes, a natural language model, and a scientific taxonomy as auxiliary information. The experiments show that the proposed model achieves $14.3 \%$ recognition accuracy for the classes with no training examples, which is significantly better than a random guess accuracy of $6.3 \%$ for 16 test classes, and three other ZSL algorithms.
\end{abstract}

Index Terms-Fine-grained classification, object recognition, zero-shot learning (ZSL).

\section{INTRODUCTION}

A DVANCES in sensor technology have increased both the spatial and the spectral resolution of remotely sensed images. Consequently, the increased resolution has exposed new details, and has enabled new object classes to be detected and recognized in aerial and satellite images.

Automatic object recognition has been one of the most popular problems in remote sensing image analysis where the algorithms aim to map visual characteristics observed in image data to object classes. Both the traditional methods

Manuscript received June 23, 2017; revised August 10, 2017; accepted September 12, 2017. Date of publication October 18, 2017; date of current version January 26, 2018. This work was supported in part by TUBITAK under Grant 116E445 and in part by the BAGEP Award of the Science Academy. (Corresponding author: Selim Aksoy.)

G. Sumbul and S. Aksoy are with the Department of Computer Engineering, Bilkent University, 06800 Ankara, Turkey (e-mail: gencer.sumbul@ bilkent.edu.tr; saksoy@cs.bilkent.edu.tr).

R. G. Cinbis was with the Department of Computer Engineering, Bilkent University, 06800 Ankara, Turkey. He is now with Middle East Technical University, 06800 Ankara, Turkey (e-mail: gcinbis@ceng.metu.edu.tr).

Color versions of one or more of the figures in this paper are available online at http://ieeexplore.ieee.org.

Digital Object Identifier 10.1109/TGRS.2017.2754648 that use various hand-crafted features with classifiers, such as support vector machines and random forests, and the more recent approaches that use deep neural networks that aim to learn both the features and the classification rules have been shown to achieve remarkable performance in data sets acquired from different sources [1], [2]. A common characteristic of such data sets in the remote sensing literature is that they contain relatively distinctive classes, with a balanced mixture of urban, rural, agricultural, and coastal land cover/use classes and object categories, for which sufficient training data to formulate a supervised learning task are often available. For example, commonly used benchmark data sets (e.g., UC Merced and AID [2]) pose the classification problem as the assignment of a test image patch to the most relevant category among the candidates, such as agricultural, beach, forest, freeway, golf course, harbor, parking lot, residential, and river. Such data sets have been beneficial in advancing the state of the art by enabling the objective comparisons of different approaches. However, the unconstrained variety of remotely sensed imagery still leads to many open problems.

A particular problem that has not received any attention in the remote sensing literature is fine-grained object recognition where one is interested in the identification of the type of an object among a large number of subcategories. Fig. 1 shows examples from the tree data set used in this paper. As seen from the 16 test classes among 40 types of street trees included in this data set, differentiating the subcategory can be a very difficult task even when very high spatial resolution image data are used. We envision that the fine-grained object recognition task will gain importance in the coming years as both the diversity and the subtleness of target object classes increase with the constantly improving spatial and spectral resolution. However, it is currently not clear how the existing classification models will behave for such recognition tasks.

Fine-grained object recognition differs from other classification and recognition tasks with respect to two important aspects: small sample size and class imbalance. Remote sensing has traditionally enjoyed the abundance of data, but obtaining label information has always been an important bottleneck in classification studies. Common attempts for reducing the effects of limited training data include regularization in parameter estimation [3] and feature extraction [4] as well as using classifier ensembles and utilizing spatial contextual information [5]. However, significantly low between-class variance and high within-class variance in fine-grained recognition tasks limit the use of such statistical solutions. Another approach for 
tackling the insufficiency of annotated samples is to use active learning for interactively collecting new examples [6], [7]. However, collecting examples for a very large number of very similar object categories in fine-grained recognition by using the visual inspection of image data can be very difficult even for domain experts, as can be seen in the aerial-view examples in Fig. 1. Furthermore, the acquisition costs for spatially distributed data can make sample collection via site visits practically unfeasible when one needs to travel unpredictably long distances to find sufficient number of examples [8]. Class imbalance in training data can also cause problems during supervised learning, particularly when the label frequencies observed in training data do not necessarily reflect the distribution of the labels among future unseen test instances.

Besides these problems, an even more extreme scenario is the zero-shot learning (ZSL) task where no training examples exists for some of the classes. To the best of our knowledge, ZSL for fine-grained object recognition has not been studied in the remote sensing literature even though it is a highly probable scenario where new object categories can be introduced after the training phase or when no training examples exist for several rare classes that are still of interest.

ZSL aims to build a recognition model for new categories that have no training examples by relating them to categories that were previously learned [9]. It is different from the domain adaptation and supervised transfer learning tasks [10] where at least some training examples are available for the target classes or the same classes exist in the target domain. Since no training instances are available for the test categories in ZSL, image data alone are not sufficient to form the association between the unseen and seen classes. Thus, we need to find new sources of auxiliary information that can act as an intermediate layer for building this association. Attributes [11], [12] have been the most popular source of auxiliary information in the computer vision literature where ZSL has recently become a popular problem [13]. Attributes often refer to the well-known common characteristics of objects, and can be acquired by human annotation. They have been successfully used in the zero-shot classification tasks for the identification of different bird or dog species or indoor and outdoor scene categories in computer vision [13]. An important requirement in the design of the attributes is that the required human effort should be small, because, otherwise, resorting to supervised or semisupervised learning algorithms by collecting training samples can be a viable alternative. An alternative is to use the automatic processing of other modalities, such as text documents [14]. As the only example in the remote sensing literature, the Word2Vec model [15] that was learned from text documents in Wikipedia was used for the zero-shot scene classification by selecting some of the scene classes in the UC Merced data set as unseen categories. New relevant attributes that exploit the peculiarities of overhead imagery should be designed for the target object categories of interest in the remotely sensed data sets.

Our main contributions in this paper are as follows. First, to the best of our knowledge, we present the first study on fine-grained object recognition with ZSL in remotely sensed imagery. The proposed approach uses a bilinear function

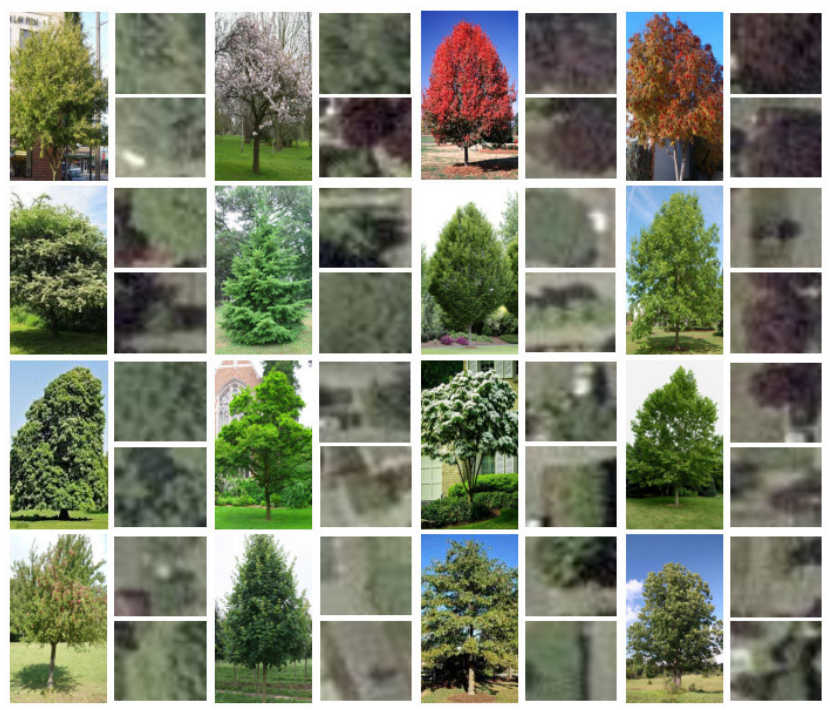

Fig. 1. Example instances for 16 classes from the fine-grained tree data set used in this paper. For each class, a ground-view photograph and two $25 \times 25$ pixel patches from aerial imagery with 1 - $\mathrm{ft}$ spatial resolution are shown. From left to right and top to bottom: London Plane, Callery Pear, Horse Chestnut, Common Hawthorn, European Hornbeam, Sycamore Maple Pacific Maple, Mountain Ash, Green Ash, Kousa Dogwood, Autumn Cherry, Douglas Fir, Orchard Apple, Apple Serviceberry, Scarlet Oak, and Japanese Snowbell.

that models the compatibility between the visual characteristics observed in the input image data and the auxiliary information that describes the semantics of the classes of interest. The image content is modeled by features extracted using a convolutional neural network (CNN) that is learned from the seen classes in the training data. The auxiliary information is gathered from three complementary domains: manually annotated attributes that reflect the domain expertise, a natural language model trained over large text corpora, and a hierarchical representation of scientific taxonomy. When the between-class variance is low and the within-class variance is high, a single source of information is often not sufficient. Thus, we exploit different representations and comparatively evaluate their effectiveness. Second, we show how the compatibility function can be estimated from the seen classes by using the maximum likelihood principle during the learning phase, and how knowledge transfer can be performed for the unseen classes by maximizing this function during the inference phase. Third, we present a new data set that contains 40 different types of trees with $1-\mathrm{ft}$ spatial resolution RGB data and point-based ground truth. We illustrate the use of this data set in a ZSL scenario by sparing some classes as unseen but it can also be used in other novel fine-grained object recognition tasks. Fourth, we present a realistic performance evaluation in a challenging setup by using different partitionings of the data, making sure that the zero-shot (unseen) categories are well isolated from the rest of the classes during both learning and parameter tuning [16].

The rest of this paper is organized as follows. Section II introduces the fine-grained data set. Section III describes the details of the methodology. Section IV presents the experiments. Section V provides the conclusions. 


\section{DATA SET}

There is currently no publicly available remote sensing data set that contains a large number of classes with high withinclass and low between-class variance. Thus, we created a new data $\operatorname{set}^{1}$ that provides a challenging test bed for finegrained object recognition research. We have gathered the data set from two main sources. The first part corresponds to point GIS data for street trees provided by the Seattle Department of Transportation in Washington State, USA [17]. In addition to location information in terms of latitude and longitude, the GIS data contain the scientific name and the common name for each tree. The second part was obtained from the Washington State Geospatial Data Archive's Puget Sound orthophotography collection [18]. This part corresponds to $1-\mathrm{ft}$ spatial resolution aerial RGB images that we mosaiced over the area covered by the GIS data.

Among the total of 126149 samples provided for 674 tree categories, we chose the top 40 categories that contain the highest number of instances. We also carefully went through every single one of the samples, and made sure that the provided coordinate actually coincides with a tree. Some samples had to be removed during this process due to mismatches with the aerial data, probably because of seasonal and temporal differences between ground-truth collection and aerial data acquisition. Finally, each tree is represented as a $25 \times 25$ pixel patch that is centered at the point ground-truth coordinate where the patch size was chosen as 25 to cover the largest tree. Overall, the resulting data set contains a total of 48063 trees from 40 different categories. The list of these categories along with the number of instances in each category is given in Table II. We use different splits of this imbalanced data set for a fair and objective evaluation of fine-grained object recognition with ZSL as suggested in [16] and presented in Section IV. Fig. 1 shows the 16 categories that are used as the unseen classes.

\section{Methodology}

In this section, we describe the mathematical formulation of our ZSL approach and the image and class representations that we utilize for describing the aerial objects and fine-grained object classes.

\section{A. Zero-Shot Learning Model}

Our goal is to learn a discriminator function that maps a given image $x \in \mathcal{X}$ to one of the target classes $y \in \mathcal{Y}$ where $\mathcal{X}$ is the space of all images and $\mathcal{Y}$ is the set of all object classes. By the definition of ZSL, training examples are available only for a subset of the classes, $\mathcal{Y}_{\text {tr }} \subset \mathcal{Y}$, which are called the seen classes. Therefore, it is not possible to directly use traditional supervised methods, such as decision trees, to build a model that can recognize the unseen classes, $\mathcal{Y}_{\text {te }} \subset \mathcal{Y}$, i.e., those with no training samples, when $\mathcal{Y}_{\mathrm{tr}} \cap \mathcal{Y}_{\mathrm{te}}=\emptyset$.

To overcome this difficulty, we first assume that a vectorspace representation, called class embedding, is available for each class. Each class embedding vector is expected to

\footnotetext{
${ }^{1}$ Available at http://www.cs.bilkent.edu.tr/ saksoy/publications.html
}

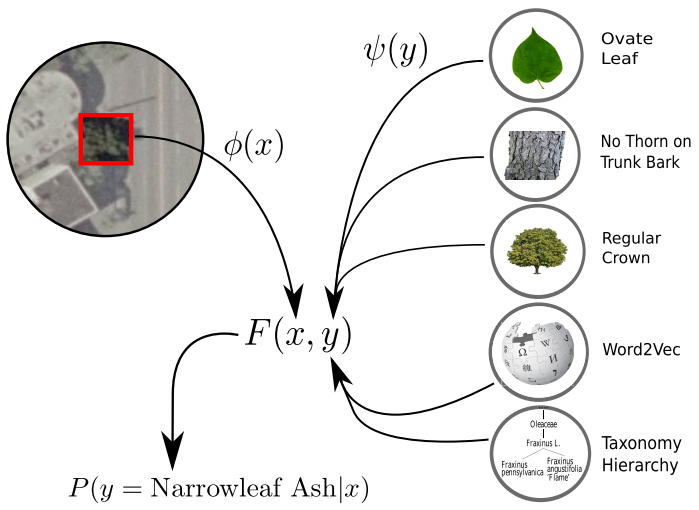

Fig. 2. Our proposed framework learns the compatibility function $F(x, y)$ between image embedding $\phi(x)$ and class embeddings $\psi(y)$ based on attributes, word embeddings from a natural language model, and a hierarchical scientific taxonomy. The learned compatibility function is then used in recognizing the instances of unseen classes by leveraging their class embedding vectors.

depict (visual) characteristics of the class, such that classification knowledge can be transferred from seen to unseen classes.

To carry out this knowledge transfer, we utilize a compatibility function $F: \mathcal{X} \times \mathcal{Y} \rightarrow \mathbb{R}$, which is a mapping from a given image-class pair $(x, y)$ to a scalar value. This value represents the confidence in assigning the image $x$ to class $y$.

Since examples only from the seen classes are available for learning the compatibility function, which will be utilized for recognizing instances of the unseen classes, $F(x, y)$ should employ a class-agnostic model. For this purpose, following the recent work on ZSL [16], we define the compatibility function in a bilinear form, as follows:

$$
F(x, y)=\phi(x)^{\top} W \psi(y) .
$$

In this equation, $\phi(x)$ is a $d$-dimensional image representation, called image embedding, $\psi(y)$ is an $m$-dimensional class embedding vector, and $W$ is a $d \times m$ matrix. This compatibility function can be considered as a class-agnostic model of a cross-domain relationship between the image representations and the class embeddings. See Fig. 2 for an illustration.

A number of empirical loss minimization schemes have been proposed for learning such ZSL compatibility functions in recent years. A detailed evaluation of these schemes can be found in [16]. In our preliminary experiments, we have investigated the state-of-the-art approaches of [9] and [14], and observed that an intuitive alternative formulation based on an adaptation of multiclass logistic regression classifier yields comparable to or better results than the others. In our approach, we define the class posterior probability distribution as the softmax of compatibility scores

$$
p(y \mid x)=\frac{\exp (F(x, y))}{\sum_{y^{\prime} \in \mathcal{Y}_{\mathrm{tr}}} \exp \left(F\left(x, y^{\prime}\right)\right)}
$$

where $\mathcal{Y}_{\text {tr }} \subset \mathcal{Y}$ is the set of seen (training) classes. Then, given $N_{\text {tr }}$ training examples, we aim to learn $F(x, y)$ using the maximum likelihood principle. Assuming that the data set contains independent and identically distributed samples, the label likelihood is given by

$$
\max _{W \in \mathbb{R}^{d \times m}} \prod_{i=1}^{N_{\mathrm{tr}}} p\left(y_{i} \mid x_{i}\right) .
$$


The optimization problem can be interpreted as finding the $W$ matrix that maximizes the predicted true class probabilities of training examples, on average. Equivalently, the parameters can be found by minimizing the negative log-likelihood

$$
\min _{W \in \mathbb{R}^{d \times m}} \sum_{i=1}^{N_{\mathrm{tr}}}-\log p\left(y_{i} \mid x_{i}\right) .
$$

To find a local optimum solution, we use stochastic gradient descent (SGD)-based optimization. The main idea in SGD is to iteratively sample a batch of training examples, compute approximate gradient over the batch, and update the model parameters using the approximate gradient. In our case, at SGD iteration $t$, the gradient matrix $G_{t}$ over a batch $B_{t}$ of training examples can be computed as follows:

$$
G_{t}=-\sum_{i \in B_{t}} \nabla_{W} \log p\left(y_{i} \mid x_{i}\right)
$$

where the gradient of the log-likelihood term for the $i$ th sample is given by

$$
\nabla_{W} \log p\left(y_{i} \mid x_{i}\right)=\phi\left(x_{i}\right) \psi\left(y_{i}\right)^{\top}-\sum_{y \in \mathcal{Y}_{\text {tr }}} p\left(y \mid x_{i}\right) \phi\left(x_{i}\right) \psi(y)^{\top} .
$$

Given the approximate gradient, the plain SGD algorithm works by subtracting a matrix proportional to $G_{t}$, from the model parameters

$$
W_{t} \leftarrow W_{t-1}-\alpha G_{t}
$$

where $W_{t}$ denotes the updated model parameters, and the learning rate $\alpha$ determines the rate of updates over the SGD iterations. It is often observed that the learning rate needs to be tuned carefully in order to avoid too large or too small parameter updates, which is necessary to maintain a stable and steady progress over the iterations. However, not only finding the right learning rate is an uneasy task, but also the optimal rate may vary across dimensions and over the iterations [19].

In order to minimize the manual effort for finding a wellperforming learning rate policy, we resort to adaptive learning rate techniques. In particular, we utilize the Adam technique [20], which estimates the learning rate for each model parameter based on the first and second moment estimates of the gradient matrix. For this purpose, we calculate the running averages of the moments at each iteration

$$
\begin{aligned}
M_{t} & =\beta_{1} M_{t-1}+\left(1-\beta_{1}\right) G_{t} \\
V_{t} & =\beta_{2} V_{t-1}+\left(1-\beta_{2}\right) G_{t}^{2}
\end{aligned}
$$

where $M_{t}$ and $V_{t}$ are the first and second moment estimates, $\beta_{1}$ and $\beta_{2}$ are the corresponding exponential decay rates, and $G_{t}^{2}$ is the elementwise square of $G_{t}$. Then, the SGD update step is modified as follows:

$$
W_{t} \leftarrow W_{t-1}-\alpha \hat{M}_{t} /\left(\sqrt{\hat{V}_{t}}+\epsilon\right)
$$

where $\hat{M}_{t}=M_{t} /\left(1-\beta_{1}^{t}\right)$ and $\hat{V}_{t}=V_{t} /\left(1-\beta_{2}^{t}\right)$ are the biascorrected first and second moment estimates. These estimates remove the inherent bias toward zero due to zero initialization of $M_{t}$ and $V_{t}$ at $t=0$, which is particularly important in early iterations. Overall, $\hat{M}_{t}$ provides a momentum-based approximation to the true gradient based on the approximate gradients over batches, and $\hat{V}_{t}$ provides a per-dimension learning rate adaptation based on an approximation to diagonal Fisher information matrix.

Finally, we should also note that we do not use an explicit regularization term on $W$ in our training formulation. Instead, we use early stopping as a regularizer. For this, we track the performance of the ZSL model on an independent validation set over optimization steps, and choose the best performing iteration. Additional details are provided in Section IV.

Once the compatibility function (i.e., the $W$ matrix) is learned, the zero-shot recognition of unseen test classes is achieved by assigning the input image to the class $y^{*}$ whose vector-space embedding yields the highest score as

$$
y^{*}=\underset{y \in \mathcal{Y}_{\mathrm{te}}}{\operatorname{argmax}} F(x, y) .
$$

In Sections III-B and III-C, we explain the details of our image representation and class embeddings, which have central importance in the ZSL performance.

\section{B. Image Embedding}

We employ a deep CNN to learn and extract region representations for aerial images. The motivation for using a CNN is to be able to exploit both the pixel-based spectral information and the spatial texture content. Spectral information available in the three visible bands is not expected to be sufficiently discriminative for fine-grained object recognition, and the learned texture representations are empirically found to be superior to hand-crafted filters.

For this purpose, based on our preliminary experiments using only the 18 seen classes from our data set (see Section IV-A for data set details), we have developed an architecture that contains three convolutional layers with $5 \times 5$, $5 \times 5$, and $3 \times 3$ dimensional filters, respectively, and two fully connected layers that map the output of the last convolutional layer to the 18 different class scores. In designing our CNN architecture, we have aimed to use filters that are large enough for learning the patterns of tree textures and shapes. We use a stride of 1 in all convolutional layers to avoid information loss, and keep the spatial dimensionality over convolutional layers via zero padding. While choosing the number of filters (64 filters per convolutional layer), we have aimed to strike the right balance between having sufficient model capacity and avoiding overfitting. We use max-pooling layers to achieve partial translation invariance [21]. Finally, we have also investigated a number of similar deeper and wider architectures, yet obtained the best performance with the presented network. Additional details of the architecture ${ }^{2}$ can be found in Fig. 3.

We train the CNN model over the seen classes using cross-entropy loss, which corresponds to maximizing the label log-likelihood in the training set. To improve training, we employ Dropout regularization [22] (with 0.9 keep probability) and Batch Normalization [23] throughout the

\footnotetext{
${ }^{2}$ While Fig. 3 shows an input with three channels, the architecture can easily be adapted to any number of input spectral bands. In general, for an input with $B$ bands, one can simply use kernels of shape $5 \times 5 \times B$ in the first layer.
} 


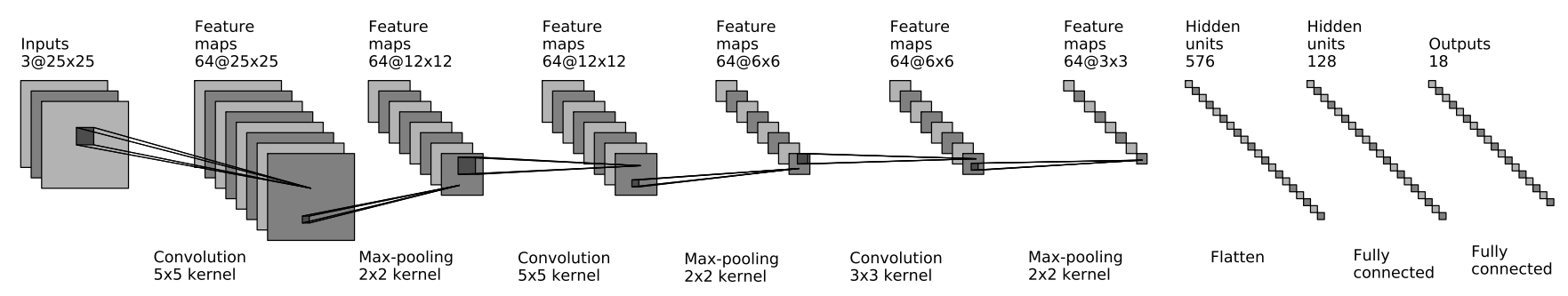

Fig. 3. Proposed deep CNN architecture with three convolutional layers containing 64 filters each with sizes $5 \times 5,5 \times 5$, and $3 \times 3$, respectively, followed by two fully connected layers containing 128 and 18 neurons, respectively. We apply max-pooling after each convolutional layer. The feature map sizes are stated at the top of each layer.

network, excluding the last layer. Once the network is trained, we use the output of the first fully connected layer, i.e., the 128-D vector shown in Fig. 3, as our image embedding $\phi(x)$ for the ZSL model. We additionally $\ell_{2}$-normalize this vector, which is a common practice for CNN-based descriptors [24].

Finally, we note that one can consider pretraining the CNN model on external large-scale data sets, such as ImageNet, and fine-tuning it to the target problem. While such an approach is likely to improve the recognition accuracy, it may also lead to biased results due to potential overlaps between the classes in our ZSL test set and the classes in the data set used during pretraining that will violate the zero-shot assumption and will hinder the objectiveness of the performance evaluation [16]. Therefore, we opt to train the CNN model solely using our own training data set.

Additional CNN training details and an empirical comparison of our CNN model to other contemporary classifiers are provided in Section IV.

\section{Class Embedding}

Class embeddings are the source of information for transferring knowledge that is relevant to classification from seen to unseen classes. Therefore, the embeddings need to capture the visual characteristics of the classes. For this purpose, following the recent work on using multiple embeddings in computer vision problems [14], we use a combination of three different class embedding methods: 1) manually annotated attributes that we collect from the target domain; 2) text embeddings generated using unsupervised language models; and 3) a hierarchical embedding based on a scientific taxonomy.

Visual attributes are obtained by determining visually distinctive features of objects, such as their parts, textures, and shapes. Since they provide a high-level description of object categories and their fine-grained properties, as perceived by humans, attributes stand out as an outstanding class embedding method for ZSL [13]. In order to utilize attributes in our work, we have collected 25 attributes for tree species, based on the Florida Trees Fact-Sheet [25]. We list the names and the possible values of these attributes in Table I. These values are encoded as binary variables in a vector.

Although attributes provide powerful class embeddings, they are typically not comprehensive in capturing the characteristics of object categories, since the attributes are defined in a manual way based on domain expertise. Our second method
TABLE I

Attributes For Fine-Grained Tree CATEgories

\begin{tabular}{|c|c|}
\hline Attribute type & Possible values \\
\hline Height (feet) & $\{10-15,15-20,20-25,25-30,30-40,40-50,50-60,60-75\}$ \\
\hline Spread (feet) & $\{10-15,15-25,25-35,35-40,40-50\}$ \\
\hline Crown uniformity & $\{$ irregular outline, regular outline $\}$ \\
\hline Crown density & \{open, moderate, dense $\}$ \\
\hline Growth rate & \{medium, fast \} \\
\hline Texture & $\{$ coarse, medium, fine $\}$ \\
\hline Leaf arrangement & \{opposite/subopposite, alternate \\
\hline Leaf shape & $\{$ ovate, star-shaped \\
\hline Leaf venation & \{palmate, pinnate \\
\hline Leaf blade length & $\{0-2,2-4,4-8\}$ \\
\hline Leaf color & \{green, purple $\}$ \\
\hline Fall color & $\{$ green, yellow, purple, red, orange $\}$ \\
\hline Fall characteristics & $\{$ not showy, showy \\
\hline Flower color & \{brown, pink, green, red, white, yellow \\
\hline Flower characteristics & $\{$ not showy, showy \\
\hline Fruit shape & \{round, elongated \\
\hline Fruit length & $\{0-0.25,0.25-0.50,0.5-1.5,1-3\}$ \\
\hline Fruit covering & $\{$ dry-hard, fleshy \\
\hline Fruit color & $\{$ brown, purple, green, red \\
\hline Fruit characteristics & $\{$ not showy, showy \\
\hline Trunk bark branches & \{no thorns, thorns\} \\
\hline Pruning requirement & $\{$ little, moderate $\}$ \\
\hline Breakage & $\{$ not resistant, resistant $\}$ \\
\hline Light requirement & $\{$ not part sun, part sun $\}$ \\
\hline Drought tolerance & $\{$ moderate, high $\}$ \\
\hline
\end{tabular}

that complements attributes is based on the unsupervised word embedding models trained over large textual corpora. For this purpose, we utilize the Word2Vec approach [15], which models the relationship between words and their contexts. Since closely related words usually appear in similar contexts, the resulting word vectors are known to implicitly encode semantic relationships. That is, words with similar meanings typically correspond to nearby locations in the embedding space. Our main goal here is to leverage the semantic relationships encoded by Word2Vec to help the ZSL model in inferring the models of unseen classes. For this purpose, we use a 1000-D embedding model trained on Wikipedia articles, and extract the word embeddings of common names of tree species (given in Table II). For categories with multiple words, we take the average of the per-word embedding vectors.

The third and the last type of class embedding that we use aims to capture the similarities across tree species based on their scientific classification. The scientific taxonomy of species in our data set is presented in Fig. 4. Since the genetics of tree species directly affect their phenotype, the taxonomic positions of trees can be informative about the visual similarity across the species. In order to capture the position and the 


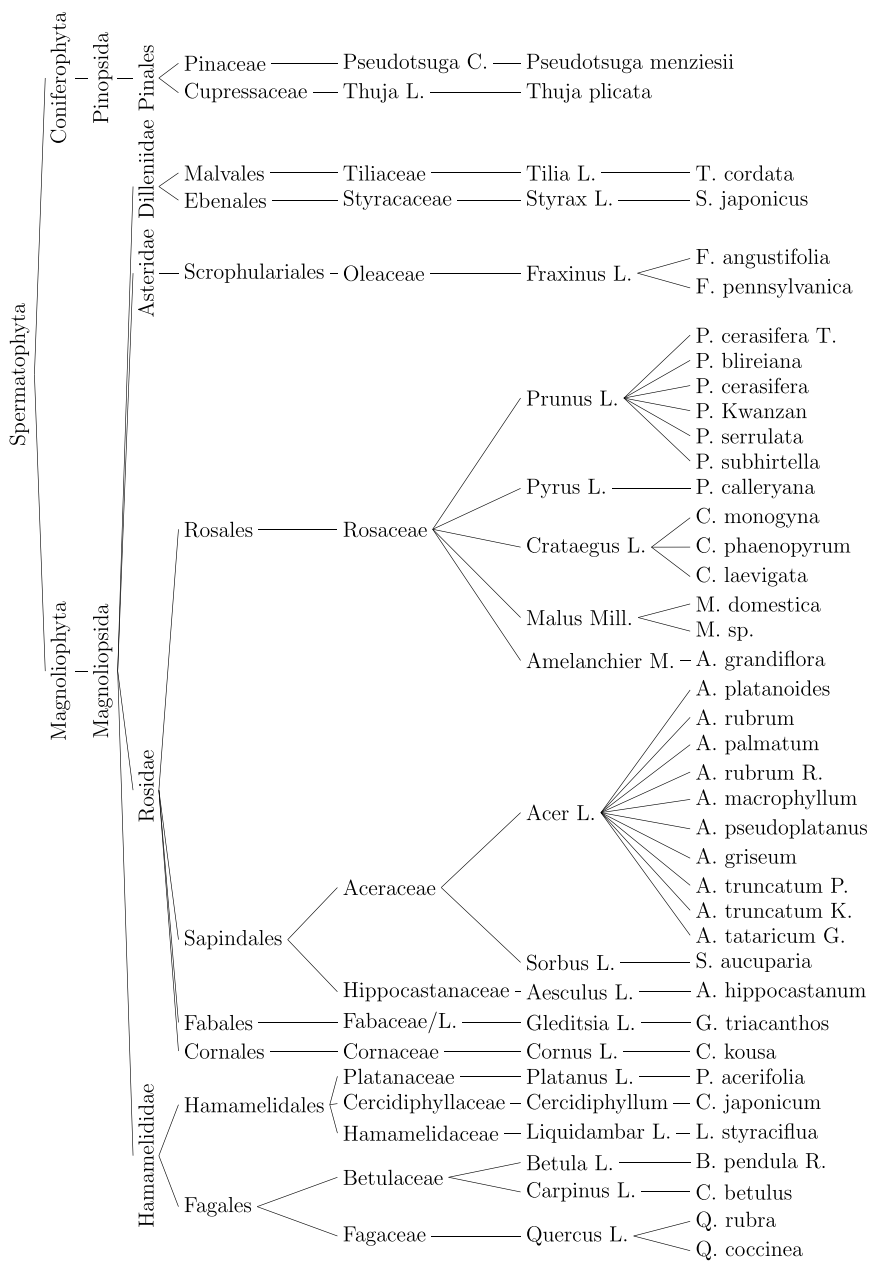

Fig. 4. Hierarchy embeddings are based on the scientific classification of tree species. This part of plant classification represents the taxonomy of our tree classes that starts with Spermatophyta superdivision and continues with the names of division, class, subclass, order, family, genus, and species in order. At each level, scientific names are written instead of common names. Classification of each tree is taken from the Natural Resources Conservation Service of the U.S. Department of Agriculture [26].

ancestors of tree species in the taxonomy tree, we apply the tree-to-vector conversion scheme described in [27]. The embedding vector corresponding to a given tree species is obtained by defining a binary value for each node in the taxonomy tree, and turning on only the values that correspond to the nodes that appear on the path from the root to the leaf node of interest. As a result, we obtain an embedding vector of length equivalent to the number of nodes in the taxonomy.

We form the final embedding vector by concatenating the vectors produced by these three embedding methods.

\section{Joint Bilinear and Linear Model}

The bilinear model specified in (1) can be interpreted as learning a weighted sum over all products of input and class embedding pairs. That is, the compatibility function can be equivalently written in the following way:

$$
F(x, y)=\sum_{u=1}^{d} \sum_{v=1}^{m} W_{u v}[\phi(x)]_{u}[\psi(y)]_{v}
$$

where $[\phi(x)]_{u}$ and $[\psi(y)]_{v}$ denote the $u$ th and $v$ th dimensions of input and class embeddings, respectively. From this interpretation, we can see that the approach can learn relations between input and class embeddings, but may not be able to evaluate the information provided by them individually. To address this shortcoming, we propose to extend the bilinear model by adding embedding-specific linear terms

$$
F_{e}(x, y)=\phi(x)^{\top} W \psi(y)+w_{x}^{\top} \phi(x)+w_{y}^{\top} \psi(y)+b
$$

where $F_{e}$ is the extended compatibility function, $w_{x}$ is the linear model over the input embeddings, $w_{y}$ is the linear model over the class embeddings, and $b$ is a bias term.

The advantage of having input and class embedding specific linear terms can be understood via the following examples: using the term $w_{x}^{\top} \phi(x)$, the model may adjust the entropy of the posterior probability distribution, i.e., the confidence in predicting a particular class, by increasing or decreasing all class scores depending on the clarity of object characteristics in the image. Similarly, using the term $w_{y}^{\top} \psi(y)$, the model can estimate a class prior based on its embedding. Finally, we note that the bias term has no effect on the estimated class posteriors given by (2), yet it simplifies the derivation given as follows.

We incorporate the linear terms of the model in a practical way by simply adding constant dimensions to both the input embedding and the class embedding. More specifically, we extend the input and class embeddings as follows:

$$
\begin{aligned}
& \phi_{e}(x)=\left[\phi(x)^{\top} 1\right]^{\top} \\
& \psi_{e}(y)=\left[\begin{array}{ll}
\psi(y)^{\top} & 1
\end{array}\right]^{\top}
\end{aligned}
$$

where $\phi_{e}(x)$ and $\psi_{e}(y)$ denote the extended embedding vectors. Similarly, we define the extended compatibility matrix $W_{e}$ as

$$
W_{e}=\left[\begin{array}{cc}
W & w_{x} \\
w_{y} & b
\end{array}\right] .
$$

It is easy to show that the bilinear product $\phi_{e}(x)^{\top} W_{e} \psi_{e}(y)$ is equivalent to the extended compatibility function $F_{e}(x, y)$, given by (8). Therefore, the linear terms can simply be introduced by adding bias dimensions to the embeddings.

\section{EXPERIMENTS}

In this section, we present a detailed experimental analysis of our approach. We first describe our experimental setup. We then present an evaluation of our CNN model in a supervised classification setting, followed by the evaluation of our ZSL approach. Finally, we experimentally analyze our model, compare it with important baselines, and discuss our findings.

\section{A. Experimental Setup}

In our experiments, we need to train and evaluate our approach in supervised and ZSL settings. Therefore, in order to obtain unbiased evaluation results, we need to define a principled way for tuning the model hyperparameters. This is particularly important in ZSL because of the expectation that the separation between the seen and unseen classes is 
clear. We follow the guidance given in [16]: 1) ZSL should be evaluated mainly on least populated classes as it is hard to obtain labeled data for fine-grained classes of rare objects; 2) hyperparameters must be tuned on a validation class split that is different from training and test classes; and 3) extracting image features via a pretrained deep neural network on a large data set should not involve zero-shot classes for training the network.

Following these guidelines, we split the 40 classes from our Seattle Trees data set into three disjoint sets (with no class overlap): 18 classes as the supervised set, 6 classes as the ZSL-validation set, and the remaining 16 classes as the ZSL-test set. The list of classes in each split is shown in Table II. We have arranged the splits roughly based on the number of examples in each class: we mostly allocated the largest classes to the supervised set, the smallest classes to the ZSL-validation set, and the remaining ones to the ZSL-test set to have a reliable performance for ZSL accuracy evaluation.

We use the supervised set for two purposes: 1) to evaluate the CNN model in a supervised classification setting and 2) to train the ZSL model using the supervised classes. For the supervised classification experiments, we use only the classes inside the supervised set, and we split the images belonging to these classes into supervised-train (60\%), supervised-validation (20\%), and supervised-test (20\%) subsets. We emphasize that these three subsets contain images belonging to the 18 supervised-set classes, and they do not contain any images belonging to a class from the ZSL-validation set or the ZSL-test set. We aim to maximize the performance on the supervised-validation set when choosing the hyperparameters of the supervised classifiers.

In the ZSL experiments, we train the ZSL model using all images from the supervised set. We use the zero-shot recognition accuracy in the ZSL-validation set for tuning the hyperparameters of the ZSL model. We evaluate the final model on the ZSL-test set, which contains the unseen classes. In this manner, we avoid using unseen classes during training or model selection, which, we believe, is fundamentally important for properly evaluating the ZSL models.

Throughout our experiments, we use normalized accuracy as the performance metric, which we obtain by averaging per-class accuracy ratios. In this manner, we aim to avoid biases toward classes with a large number of examples.

\section{B. Supervised Fine-Grained Classification}

Before presenting our ZSL results, we first evaluate our $\mathrm{CNN}$ model in a supervised setting to compare it against other mainstream supervised classification techniques, and to give a sense of the difficulty of the fine-grained classification problem that we propose. For this purpose, we use logistic regression and random forest classifiers as our baselines. For a fair comparison, we train all methods on the supervisedtrain set, and tune their hyperparameters on the supervisedvalidation set.

We train our CNN architecture using SGD with the Adam method [20] that we also use for the ZSL model estimation as described in Section III-A. Based on the supervised-validation
TABLE II

Class Separation Used for the Data Set and the Number of INSTANCES IN EACH Class

\begin{tabular}{|c|c|c|}
\hline Supervised-set & $\begin{array}{c}\text { ZSL- } \\
\text { validation }\end{array}$ & ZSL-test \\
\hline 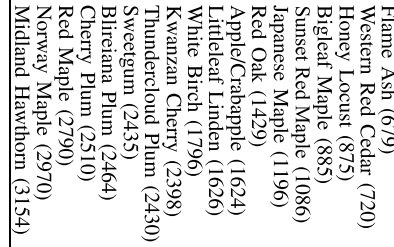 & $\begin{array}{ll} \\
0 \\
0\end{array}$ & 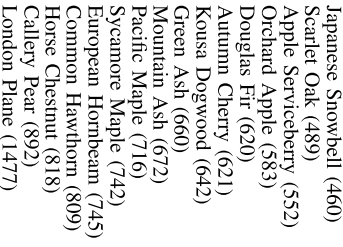 \\
\hline
\end{tabular}

TABLE III

SUPERVISED CLASSIFICATION RESULTS (IN \%)

\begin{tabular}{lccccc}
\cline { 2 - 5 } & $\begin{array}{c}\text { Random } \\
\text { guess }\end{array}$ & $\begin{array}{c}\text { Logistic } \\
\text { regression }\end{array}$ & $\begin{array}{c}\text { Random } \\
\text { forest }\end{array}$ & CNN & $\begin{array}{c}\text { CNN with } \\
\text { perturbation }\end{array}$ \\
\hline $\begin{array}{c}\text { Normalized } \\
\text { accuracy }\end{array}$ & 5.6 & 16.4 & 15.7 & 27.9 & 34.6 \\
\hline
\end{tabular}

set, we have set the initial learning rate of Adam to $10^{-3}$, the mini-batch size to 100 , and the $\ell_{2}$-regularization weight to $10^{-5}$. We also observed that it is beneficial to add the perturbations of training examples by randomly shifting each region with an amount in the range from zero to $20 \%$ of the height/width.

We compare the resulting classifiers on the supervised-test set, as shown in Table III. From these results, we can see that all classification methods perform clearly better than the random guess baseline (5.6\%). In addition, we can see that the proposed CNN model both without perturbation (27.9\%) and with perturbation (34.6\%) outperforms logistic regression $(16.4 \%)$ and random forest $(15.7 \%)$ by a large margin.

These results highlight the advantage of the deep image representation learned by the $\mathrm{CNN}$ approach. In addition, we can observe the difficulty of the fine-grained classification problem, which is quite different from the traditional classification scenarios that aim to discriminate buildings from trees or roads from grass. We believe that the fine-grained classification is an important open problem in remote sensing, and can lead to advances in object recognition research.

\section{Fine-Grained Zero-Shot Learning}

In this section, we evaluate our ZSL approach and compare against three state-of-the-art ZSL methods: embarrassingly simple ZSL (ESZSL) [9], structured label embedding (SJE) [14], and attribute label embedding (ALE) [28]. We train all ZSL models over the supervised-train set, and tune all model hyperparameters according to normalized accuracy on the ZSL-validation set.

For our approach, we initialize the $W$ matrix randomly from a uniform distribution [29] and train the model using Adam optimizer [20]. We tune the hyperparameters of initial learning rate of Adam and the number of training iterations (for early stopping-based regularization). For the SJE [14] and ALE [28] baselines, we use SGD for training. Unlike the original papers that use a constant learning rate for SGD, we have found that 
TABLE IV

ZSL RESULTS (IN \%)

\begin{tabular}{lccccc}
\cline { 2 - 5 } & $\begin{array}{c}\text { Random } \\
\text { guess }\end{array}$ & ALE [28] & SJE [14] & ESZSL [9] & Ours \\
\hline $\begin{array}{c}\text { Normalized } \\
\text { accuracy }\end{array}$ & 6.3 & 12.5 & 12.6 & 13.2 & 14.3 \\
\hline
\end{tabular}

decreasing the learning rate regularly over epochs leads to better performance for these baselines. We tune the learning rate policy on the ZSL-validation set. For the ESZSL [9] baseline, we tune its regularization parameters $\lambda$ and $\gamma$ by choosing the best-performing combination of the parameters in the range $\left\{10^{-3}, 10^{-2}, 10^{-1}, 10^{0}, 10^{1}, 10^{2}, 10^{3}\right\}$ according to the ZSL-validation set and fix the $\beta$ hyperparameter to $\lambda \gamma$, as suggested in [9]. In this case, the optimal compatibility matrix is given by a closed-form solution [9]. Finally, we note that all compared methods learn a single compatibility $W$ matrix, which provides a fair comparison across them.

For all methods, we have observed that imbalance in terms of the number of examples across the training classes can negatively affect the resulting ZSL model. To alleviate this problem, we apply random oversampling to the training set, such that the size of the training set for each class is equivalent to the size of the largest class.

The ZSL results over the 16 ZSL-test classes are presented in Table IV. Our ZSL model achieves a $14.3 \%$ normalized accuracy, which is clearly better than the random guess baseline (6.3\%), ALE (12.5\%), SJE (12.6\%), and ESZSL (13.2\%). These results validate the effectiveness of our probabilistic ZSL formulation.

The image embedding can have a profound effect on the ZSL performance. To better understand the efficacy of our representation, we train our ZSL model over the outputs of different CNN layers (Fig. 3), and tune the number of training iterations on ZSL-validation for each one separately. When we use the 18-D classification outputs, the ZSL performance drops from $14.3 \%$ to $8.5 \%$. Similarly, if we use the outputs of the layers preceding the first fully connected layer, the performance drops from $14.3 \%$ to $13.0 \%$ for the last maxpooling output (equivalently, the Flatten output), to $12.8 \%$ for the convolutional layer with $3 \times 3$ kernels, and to $11.1 \%$ for the preceding max-pooling output. Simply using the original RGB image results in $8.3 \% \mathrm{ZSL}$ performance. Overall, these results highlight the importance of the image representation on the ZSL performance, and suggest that the fully connected layer preceding the classification layer results in relatively generic features that are suitable for ZSL, in our architecture.

Our class embedding is a combination of three different embedding techniques. To understand the contribution of each one, we present the ZSL performance for each possible combination of class embedding methods in Table V. The first three rows of Table $\mathrm{V}$ indicate that when the embedding techniques are used individually, they result in a comparable performance, with a higher performance for the Word2Vec (12.1\%), compared with attributes $(8.4 \%)$ and hierarchy $(9.7 \%)$. The following three rows indicate that the hierarchy-Word2Vec (13.2\%) embedding pair leads to better results compared with indi-
TABLE V

EFFECT OF DIFFERENT CLASS EMBEDDINGS ON ZSL PERFORMANCE (IN \%)

\begin{tabular}{cccc}
\hline Attribute & Hierarchy & Word2Vec & $\begin{array}{c}\text { Normalized } \\
\text { accuracy }\end{array}$ \\
\hline$\checkmark$ & $x$ & $x$ & 8.4 \\
$x$ & $\checkmark$ & $x$ & 9.7 \\
$x$ & $x$ & $\checkmark$ & 12.1 \\
$\checkmark$ & $\checkmark$ & $x$ & 11.2 \\
$\checkmark$ & $x$ & $\checkmark$ & 12.6 \\
$x$ & $\checkmark$ & $\checkmark$ & 13.2 \\
$\checkmark$ & $\checkmark$ & $\checkmark$ & $\mathbf{1 4 . 3}$ \\
\hline
\end{tabular}

TABLE VI

EFFECT OF Linear TERMS ON ZSL PERFormance (IN \%)

\begin{tabular}{ccc}
\hline $\begin{array}{c}\text { For image } \\
\text { embedding }\end{array}$ & $\begin{array}{c}\text { For class } \\
\text { embedding }\end{array}$ & $\begin{array}{c}\text { Normalized } \\
\text { accuracy }\end{array}$ \\
\hline$x$ & $x$ & 11.8 \\
$\checkmark$ & $x$ & 12.2 \\
$x$ & $\checkmark$ & 13.4 \\
$\checkmark$ & $\checkmark$ & $\mathbf{1 4 . 3}$ \\
\hline
\end{tabular}

vidual embeddings as well as the pairs of attribute-Word2 Vec $(12.6 \%)$ or hierarchy-attribute $(11.2 \%)$. These results show that our hierarchy and Word2Vec embeddings are more effective than attribute embeddings. This observation suggests that the recognition accuracy can be improved possibly by defining more descriptive attributes. On the other hand, the final result based on the combination of all embeddings, which leads to the highest accuracy (14.3\%), shows that our class embeddings are complementary to each other.

Another important aspect of the proposed method is extending the bilinear model by adding linear terms for the input and class embeddings. To understand the significance of this extension, we present an evaluation of the linear terms in Table VI. Table VI shows that without having any linear term, the normalized accuracy for ZSL is $11.8 \%$. Adding $w_{x}^{\top} \phi(x)$ [see (8)] improves the performance to $12.2 \%$, and adding $w_{y}^{\top} \psi(y)$ improves the performance to $13.4 \%$. Finally, adding both terms together leads to our highest result of $14.3 \%$ normalized accuracy. These results validate the importance of adding linear terms into the bilinear ZSL model.

\section{Analysis and Discussion}

The results presented so far show that the proposed ZSL approach performs significantly better than the random guess baseline, and also better than several other state-of-the-art ZSL methods. However, an important question is how well the ZSL performs in a practical sense. To address this question, we compare our ZSL approach against supervised classification, and discuss the relative advantages and disadvantages of supervised learning versus ZSL of novel class models.

For this purpose, we use fivefold cross validation over the whole ZSL-test set, where repeatedly one of the folds is utilized for training the supervised classifiers, and the remaining folds are utilized as the test subset. In our analysis, we consider two types of supervised classifiers: 1) CNN models that are 


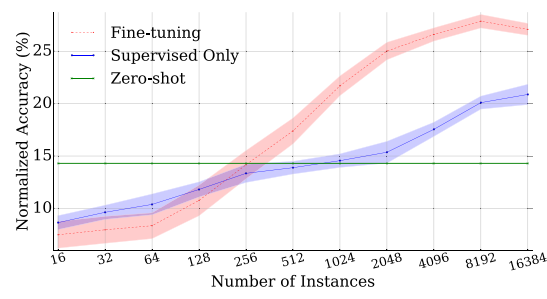

Fig. 5. Performance comparison of the proposed framework with finetuning and supervised-only methods on zero-shot test classes. Fine-tuning and supervised-only results are derived at different points when the number of instances is increasing. The $x$-axis is shown in log scale.

trained from scratch over supervised ZSL-test examples only and 2) pretrained CNN models that are fine-tuned to the ZSL-test classes. For the latter approach, we reinitialize and retrain the last layer, i.e., the classification layer, of our pretrained CNN model. Our motivation for fine-tuning is that all layers preceding the last layer are likely to extract a classagnostic image representation, and the last layer can be interpreted as a linear classifier that transforms the learned image representation into the classification scores. In this way, we can effectively transfer knowledge from the supervised set to the ZSL-test set, using supervised training examples for the latter.

It is well known that the accuracy of a supervised classifier tends to improve as its training set gets larger. In this context, to understand the tradeoff between using a ZSL approach, which uses zero training examples for the target classes, versus collecting supervised training examples, we train the supervised classifiers at varying number of training examples. More specifically, we train separate supervised classifiers by limiting the number of examples per class to each possible constant in $1,2,4, \ldots, 2^{10}$. We impose these limits by subsampling the training subset at each fold of fivefold cross validation. To obtain reliable statistics, we repeat each experiment ten times.

Fig. 5 presents the results for the supervised-only CNN, fine-tuned CNN, and the ZSL model. The $x$-axis shows the number of training examples for the supervised classifiers, and the $y$-axis shows the corresponding normalized accuracy scores. The curves are obtained by averaging results over all folds and all runs, and setting the curve thickness to the standard deviation of the results. The ZSL approach is shown as a single horizontal line, as it does not use any supervised training examples.

From the results, we can see that the supervised-only CNN starts to match the ZSL performance only when the number of training examples is more than 512, and the fine-tuned CNN reaches the ZSL performance at 256 samples. This is a significant achievement considering that: 1) the ZSL approach uses zero training examples from the target classes and 2) we are working with fine-grained categories that are hard to distinguish even by visual inspection of the image data. We expect the ZSL performance to further improve following the advances in image representation, image resolution, class embeddings, and ZSL formulations.

Importantly, we should also note that the collection and the annotation of even 256 training examples can be a very costly task: sample collection may require spatially surveying a very

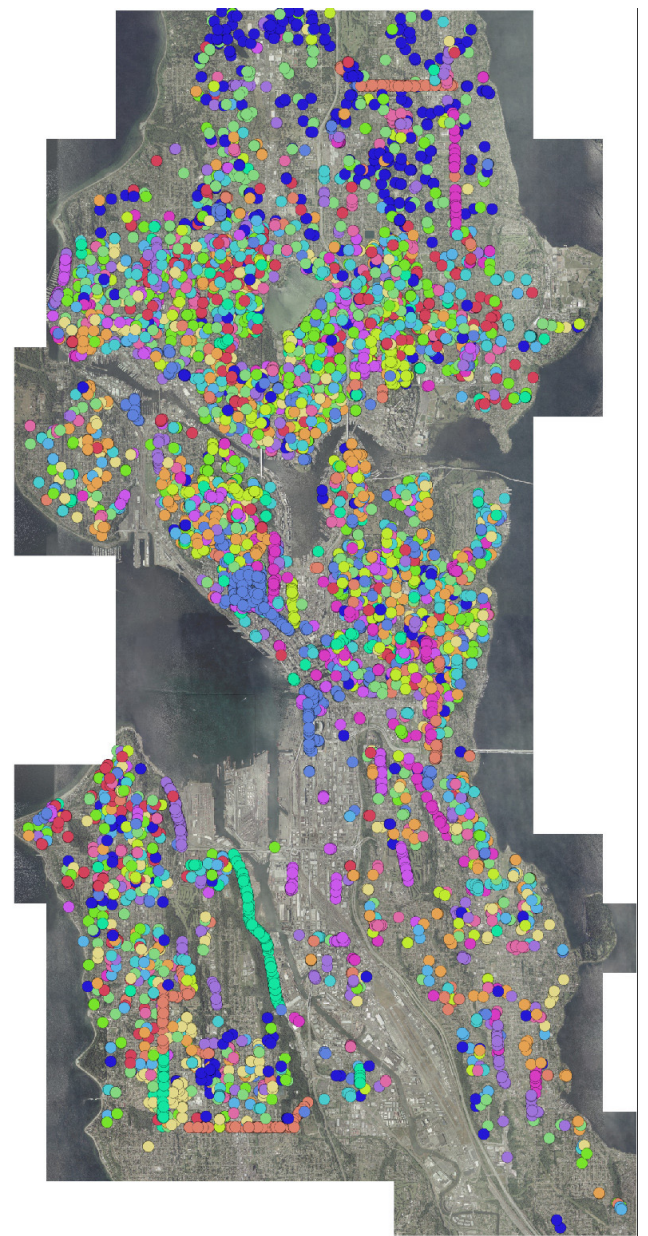

Fig. 6. Spatial distribution of instances belonging to the zero-shot test (unseen) classes. Each point shows one instance, and the point colors represent the classes. (Best viewed in color.)

large area, and annotating them with class labels typically requires the inspection of the instances or their close-by pictures by domain experts, as the most fine-grained categories are very difficult to distinguish. For example, Fig. 6 shows the Seattle region and the spatial distribution of our 16-class ZSL-test instances in this region. In Fig. 6, we observe that the instances and the classes are scattered all around an area of $217 \mathrm{~km}^{2}$, which casts the data collection and annotation a very time-consuming and challenging task. In this context, we believe that ZSL of fine-grained categories can potentially become a central topic toward building semantically rich image understanding systems for remote sensing.

\section{CONCLUSION}

We studied the ZSL problem for fine-grained object recognition in remotely sensed images. To cope with the difficulty of learning a very large number of very similar object categories as well as the need for being able to recognize classes even when there are no training examples, our framework exploited alternative sources of auxiliary information to build an association between the seen and unseen classes. The proposed approach learned a bilinear function from the seen classes, so that the compatibility between the visual characteristics 
observed in the input image data and the auxiliary information that described the semantics of the classes of interest is modeled. Then, we showed how this compatibility function could be used for performing knowledge transfer during the inference of the unseen classes. Extensive experiments using different partitionings of a challenging aerial data set with 40 types of street trees defined as fine-grained target classes showed that our method obtained $14.3 \%$ classification accuracy, which was significantly better than random guessing $(6.3 \%)$ for 16 test classes and three other ZSL algorithms from the literature. Future work includes new representations for auxiliary information that models different aspects of spectral and spatial data characteristics as well as the domainspecific class semantics.

\section{REFERENCES}

[1] F. Hu, G.-S. Xia, J. Hu, and L. Zhang, "Transferring deep convolutional neural networks for the scene classification of high-resolution remote sensing imagery," Remote Sens., vol. 7, no. 11, pp. 14680-14707, Nov. 2015.

[2] G.-S. Xia et al., "AID: A benchmark data set for performance evaluation of aerial scene classification," IEEE Trans. Geosci. Remote Sens., vol. 55, no. 7, pp. 3965-3981, Jul. 2017.

[3] B.-C. Kuo and D. A. Landgrebe, "A covariance estimator for small sample size classification problems and its application to feature extraction," IEEE Trans. Geosci. Remote Sens., vol. 40, no. 4, pp. 814-819, Apr. 2002.

[4] B.-C. Kuo and K.-Y. Chang, "Feature extractions for small sample size classification problem," IEEE Trans. Geosci. Remote Sens., vol. 45, no. 3, pp. 756-764, Mar. 2007

[5] F. Li, L. Xu, P. Siva, A. Wong, and D. A. Clausi, "Hyperspectral image classification with limited labeled training samples using enhanced ensemble learning and conditional random fields," IEEE J. Sel. Topics Appl. Earth Observ. Remote Sens., vol. 8, no. 6, pp. 2427-2438, Jun. 2015

[6] D. Tuia, F. Ratle, F. Pacifici, M. F. Kanevski, and W. J. Emery, "Active learning methods for remote sensing image classification," IEEE Trans. Geosci. Remote Sens., vol. 47, no. 7, pp. 2218-2232, Jul. 2009.

[7] B. Demir, C. Persello, and L. Bruzzone, "Batch-mode active-learning methods for the interactive classification of remote sensing images," IEEE Trans. Geosci. Remote Sens., vol. 49, no. 3, pp. 1014-1031, Mar. 2011.

[8] A. Loi, G. Jun, and J. Ghosh, "Active learning of hyperspectral data with spatially dependent label acquisition costs," in Proc. IEEE Int. Geosci. Remote Sens. Symp., Jul. 2009, pp. V-256-V-259.

[9] B. Romera-Paredes and P. Torr, "An embarrassingly simple approach to zero-shot learning," in Proc. Int. Conf. Mach. Learn., vol. 37. 2015, pp. 2152-2161.

[10] D. Tuia, C. Persello, and L. Bruzzone, "Domain adaptation for the classification of remote sensing data: An overview of recent advances," IEEE Geosci. Remote Sens. Mag., vol. 4, no. 2, pp. 41-57, Jun. 2016.

[11] V. Ferrari and A. Zisserman, "Learning visual attributes," in Proc. Adv. Neural Inf. Process. Syst., 2007, pp. 433-440.

[12] A. Farhadi, I. Endres, D. Hoiem, and D. Forsyth, "Describing objects by their attributes," in Proc. IEEE Conf. Comput. Vis. Pattern Recognit., Jun. 2009, pp. 1778-1785.

[13] C. H. Lampert, H. Nickisch, and S. Harmeling, "Attribute-based classification for zero-shot visual object categorization," IEEE Trans. Pattern Anal. Mach. Intell., vol. 36, no. 3, pp. 453-465, Mar. 2014

[14] Z. Akata, S. Reed, D. Walter, H. Lee, and B. Schiele, "Evaluation of output embeddings for fine-grained image classification," in Proc. IEEE Conf. Comput. Vis. Pattern Recognit., Jun. 2015, pp. 2927-2936.

[15] T. Mikolov, I. Sutskever, K. Chen, G. S. Corrado, and J. Dean, "Distributed representations of words and phrases and their compositionality," in Proc. Adv. Neural Inf. Process. Syst., 2013, pp. 3111-3119.

[16] Y. Xian, B. Schiele, and Z. Akata, "Zero-shot learning-the good, the bad and the ugly," in Proc. IEEE Conf. Comput. Vis. Pattern Recognit., to be published.

[17] Seattle Department of Transportation, Seattle, WA, USA. (Oct. 2016) Seattle Street Trees. Accessed: Oct. 2106. [Online]. Available: http://web6.seattle.gov/SDOT/StreetTrees/
[18] Washington State Geospatial Data Archive. (Oct. 2016). Puget Sound Orthophotography. [Online]. Available: https://wagda.lib.washington. edu/data/type/photography/puget_sound/

[19] I. Goodfellow, Y. Bengio, and A. Courville, Deep Learning. Cambridge, MA, USA: MIT Press, 2016. [Online]. Available: http://www.deeplearningbook.org

[20] D. P. Kingma and J. Ba, "Adam: A method for stochastic optimization," in Proc. Int. Conf. Learn. Represent., May 2015, pp. 1-15.

[21] D. Mishkin, N. Sergievskiy, and J. Matas, "Systematic evaluation of convolution neural network advances on the ImageNet," Comput. Vis. Image Understand., vol. 161, pp. 11-19, Aug. 2017.

[22] N. Srivastava, G. Hinton, A. Krizhevsky, I. Sutskever, and R. Salakhutdinov, "Dropout: A simple way to prevent neural networks from overfitting," J. Mach. Learn. Res., vol. 15, no. 1, pp. 1929-1958, 2014.

[23] S. Ioffe and C. Szegedy, "Batch normalization: Accelerating deep network training by reducing internal covariate shift," in Proc. Int. Conf. Mach. Learn., 2015, pp. 448-456.

[24] A. S. Razavian, H. Azizpour, J. Sullivan, and S. Carlsson, "CNN features off-the-shelf: An astounding baseline for recognition," in Proc. IEEE Conf. Comput. Vis. Pattern Recognit., Jun. 2014, pp. 806-813.

[25] University of Florida, Gainesville, FL, USA. (Oct. 2016). 680 Tree Fact Sheets: Trees by Common Name. [Online]. Available: http://hort.ifas.ufl. edu/database/trees/trees_common.shtml

[26] U.S. Department of Agriculture, Natural Resources Conversation Service, Washington, DC, USA. Plants Database. Accessed: Oct. 2016 [Online]. Available: https://plants.usda.gov/java/

[27] R. Mittelman, M. Sun, B. Kuipers, and S. Savarese, "A Bayesian generative model for learning semantic hierarchies," Frontiers Psychol., vol. 5, p. 417, May 2014.

[28] Z. Akata, F. Perronnin, Z. Harchaoui, and C. Schmid, "Label-embedding for image classification," IEEE Trans. Pattern Anal. Mach. Intell., vol. 38, no. 7, pp. 1425-1438, Jul. 2016

[29] X. Glorot and Y. Bengio, "Understanding the difficulty of training deep feedforward neural networks," in Proc. Int. Conf. Artif. Intell. Statist., 2010, pp. 249-256.

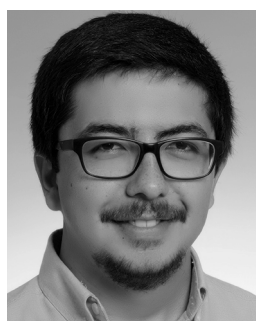

Gencer Sumbul received the B.S. degree in computer engineering from Bilkent University, Ankara, Turkey, in 2015 , where he is currently pursuing the M.S. degree in computer engineering.

His research interests include computer vision, deep learning, and machine learning, with special interest in fine-grained object recognition with weak supervision on remote sensing images.

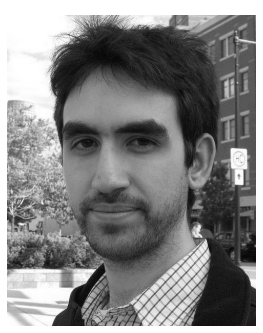

Ramazan Gokberk Cinbis received the B.S. degree from Bilkent University, Ankara, Turkey, in 2008 , and the M.A. degree from Boston University, Boston, MA, USA, in 2010. He was a doctoral student in the LEAR team, INRIA Grenoble between 2010-2014, and received the Ph.D. degree in computer science from Université de Grenoble, Grenoble, France, in 2014

$\mathrm{He}$ is currently an Assistant Professor with Middle East Technical University, Ankara. His research interests include computer vision and machine learning, with special interest in statistical image models, and deep learning with weak supervision.

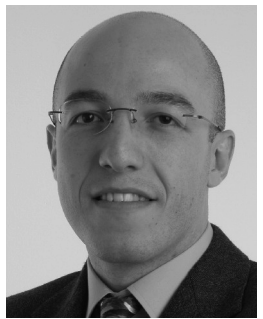

Selim Aksoy (S'96-M'01-SM'11) received the B.S. degree from Middle East Technical University, Ankara, Turkey, in 1996, and the M.S. and Ph.D. degrees from the University of Washington, Seattle, WA, USA, in 1998 and 2001, respectively.

$\mathrm{He}$ is currently an Associate Professor with the Department of Computer Engineering, Bilkent University, Ankara. His current research interests include computer vision, statistical and structural pattern recognition, and machine learning. 